\title{
La aspirina en la prevención primaria del cáncer colorrectal
}

\section{Aspirin in the primary prevention of colorectal cancer}

\author{
M. Fernández-Calderón, M.T. Betés Ibáñez
}

\section{RESUMEN}

La aspirina y otros antiinflamatorios no esteroideos (AINE) se han asociado a una reducción en el riesgo de desarrollar adenomas y cáncer colorrectal. Con la evidencia científica disponible, queda justificada la necesidad de más estudios que evalúen el efecto protector de la aspirina u otros AINE como medida preventiva del cáncer de colon, que definan la dosis mínima eficaz, la edad a la que comenzar el tratamiento, la duración más conveniente del mismo y las subpoblaciones para las que los beneficios de la quimioprofilaxis superan los posibles efectos adversos.

Palabras clave. Aspirina. Cáncer colorrectal. Prevención.

\begin{abstract}
Aspirin and other non-steroidal anti-inflammatory drugs (NSAIDs) have been associated with a reduction in the risk of developing adenomas and colorectal cancer. The available scientific evidence justifies the need for more studies that evaluate the protective effect of aspirin and other NSAIDs as a preventive measure against cancer of the colon; define the minimum efficient dosage; the age at which to begin treatment; the most convenient duration of the latter; and the sub-populations for which the benefits of chemoprophylaxis outweigh possible adverse effects.
\end{abstract}

Key words. Aspirin. Colorectal cancer. Prevention.
Departamento de Digestivo

Clínica Universidad de Navarra

Pamplona

Recepción: 13 de febrero de 2012

Aceptación provisional: 23 de febrero de 2012

Aceptación definitiva: 23 de febrero de 2012

\section{Correspondencia}

M. Fernández Calderón

Departamento de Digestivo

Clínica Universidad de Navarra

Avda. Pío XII, 36

31008 Pamplona

E-mail: mfdezcalderon@gmail.com 


\section{INTRODUCCIÓN}

El cáncer colorrectal (CCR) ocupa el $2^{\circ}$ lugar como causa de mortalidad por cáncer, con 492.000 muertes por año en todo el mundo. Se ha estimado que aproximadamente un $6 \%$ de las personas desarrollarán esta neoplasia a lo largo de su vida. Según los datos disponibles, en España también constituye la $2^{\mathrm{a}}$ causa de muerte por cáncer, siendo responsable del $12 \%$ de las defunciones por enfermedad neoplásica en varones y del $15 \%$ en mujeres en $2006^{1}$.

Aunque se han establecido diferentes grupos de riesgo que cada vez están mejor definidos (cáncer colorrectal hereditario y familiar $^{2}$ ), hasta un $70 \%$ de estos cánceres se diagnostican en sujetos que no pertenecen a dichos grupos; este hecho, junto a la alta frecuencia con que se diagnostica la enfermedad en nuestro medio, hace que los programas de prevención primaria sean especialmente atractivos en el CCR.

Los programas de prevención primaria pretenden evitar el desarrollo de enfermedades. Esto supone la identificación y modificación de los agentes etiológicos de la enfermedad. En la patogenia del CCR, además de factores genéticos, no modificables, se acepta que los factores ambientales cumplen un factor fundamental. Basados en la evidencia científica de que disponemos actualmente, se han establecido diversas recomendaciones para la prevención primaria del CCR en la población general ${ }^{3}$. Una forma de prevención primaria que actualmente constituye una línea de investigación en auge es la utilización de agentes farmacológicos, también llamada quimioprofilaxis. La aspirina (AAS) y otros AINE han sido los fármacos más ampliamente estudiados en este campo.

\section{MECANISMOS ANTICARCINOGÉNICOS DE LOS AINE}

La base biológica que explica las propiedades anticarcinogénicas de los AINE viene dada por dos mecanismos: su capacidad de inducir apoptosis incrementando sustancialmente el ácido araquidónico, precursor de prostaglandinas; y su acción inhibitoria sobre la ciclooxigenasa (COX), enzima con acción carcinógena, mediada por prostaglandinas. Se conocen 2 isoformas de la COX: la COX-1, presente en la mayoría de los tejidos y a la que se atribuye un papel regulador en la homeostasis fisiológica de diferentes órganos y tejidos; y la COX-2, prácticamente indetectable en la mayoría de los tejidos y que se induce por citoquinas, mitógenos y factores de crecimiento $^{4}$. Estudios recientes han observado niveles altos de prostaglandinas y COX-2 en adenomas y tumores colorrectales, en comparación con tejido normal. Se ha visto que las prostaglandinas están asociadas con la angiogénesis tumoral, la proliferación celular, inhibición de la vigilancia inmune y la apoptosis ${ }^{5}$. La expresión de COX-2 se ha relacionado con el tamaño y el grado de displasia del adenoma, por lo que fármacos inhibidores selectivos de la COX-2 podrían tener un efecto protector en la aparición de adenomas y, por tanto, de $\mathrm{CCR}^{6}$. Además se conoce que los AINE inhiben la angiogénesis y pueden modular las vías carcinogénicas relacionadas con la insulina, pero continuamos sin conocer otros mecanismos que puedan tener relevancia clínica ${ }^{5}$.

\section{EFECTO QUIMIOPROTECTOR DE LOS AINE}

La mayoría de la evidencia científica disponible sobre el efecto quimioprotector de la AAS u otros AINE (incluyendo los inhibidores selectivos de la COX-2), proviene de estudios observacionales en los que se demuestra que el uso regular de estos fármacos, reduce en un $20-40 \%$ el riesgo de desarrollo de adenomas de colon y de CCR en individuos de riesgo medio ${ }^{7}$. Dado que la mayoría de los CCR surgen a partir de lesiones preneoplásicas como son los adenomas, éstos se han convertido en el punto de mira para muchos de los ensayos clínicos que pretenden hacer prevención en CCR.

La primera vez que se empezó a observar una asociación sólida entre el uso de aspirina y un menor riesgo de CCR y ade- 
nomas, fue en la década de los años 80 y principios de los 90 , en los que se realizaron varios estudios de casos y controles que fueron seguidos de estudios prospectivos de cohortes. Teniendo en cuenta que los pacientes con historia previa de CCR se podrían considerar "a priori" como un grupo de mayor riesgo para el desarrollo de adenomas, se empezó a estudiar el posible efecto quimioprotector de la AAS en este subgrupo de pacientes. Cuatro ensayos aleatorizados de AAS frente a placebo ${ }^{8-11}$, evidenciaron que la AAS inhibía la carcinogénesis y reducía el riesgo de adenomas de colon en pacientes con historia previa de CCR. Un meta-análisis reciente de estos 4 ensayos ha demostrado que los pacientes consumidores de AAS tienen un RR combinado de 0,83 de desarrollar adenomas (IC 95\% 0,72-0,96) y un RR combinado de 0,72 de desarrollar adenomas avanzados (IC 95\% 0,57-0,90), es decir una reducción global del riesgo cercana al $30 \%{ }^{12}$. Sin embargo las dosis de AAS y los periodos de seguimiento difieren mucho de un estudio a otro y aunque efectivamente se observa un efecto protector, no se pueden extraer conclusiones unánimes.

No todos los estudios muestran un efecto quimioprotector. Hay 2 ensayos clínicos aleatorizados (The Women's Health Study y The Physicians'Health Study) que han evaluado el efecto de la AAS sobre la incidencia de cáncer en población sana no perteneciente a grupos de alto riesgo. En el primero se aleatorizó a 39.876 mujeres sanas, profesionales sanitarias, menores de 45 años, a recibir $100 \mathrm{mg}$ de AAS o placebo a diario durante 10 años, observando que la aspirina no tenía efecto en la incidencia de ningún tipo de cáncer en particular ${ }^{13}$. En el segundo, se aleatorizó a 22.071 varones médicos a recibir $325 \mathrm{mg}$ de AAS o placebo a días alternos, sin encontrar una reducción en la incidencia de CCR en el grupo de tratamiento después de 5 años de seguimiento ${ }^{14}$. Estos hallazgos se confirmaron en un análisis posterior, a 12 años de seguimiento ${ }^{15}$.

Quizá uno de los trabajos más interesantes ha sido recientemente publicado por el grupo de Rothwell y col $^{16}$ que ana- liza 5 ensayos randomizados y controlados diseñados inicialmente para estudiar la prevención con aspirina de episodios adversos cardiovasculares. Este trabajo engloba a más de 14.000 pacientes y evalúa el efecto de la AAS sobre la incidencia y la mortalidad por CCR durante un periodo de seguimiento largo (más de 20 años). Se demuestra que efectivamente el efecto de la AAS depende de la dosis y protege a bajas dosis $(75 \mathrm{mg})$ consiguiendo una reducción de hasta un $25 \%$ en la incidencia de CCR y del 35\% en la mortalidad por CCR. Dosis mayores (> $300 \mathrm{mg}$ ) no aportan más y favorecen la aparición de efectos secundarios. Sin embargo, estos estudios no estaban dirigidos específicamente al estudio del CCR, no se registraron los efectos secundarios de la AAS y cabe considerar un posible sesgo por sangrado (ya que de esta forma se puede manifestar precozmente la enfermedad)

También se han realizado estudios en pacientes de alto riesgo. Por ejemplo, el grupo de investigación CAPP2 (Colorectal Adenoma-carcinoma Prevention Programme 2), evaluó por medio de un ensayo clínico el efecto de la aspirina sobre el riesgo de CCR y cáncer de endometrio en pacientes con Síndrome de Lyncha, demostrando que la AAS no tiene un efecto quimioprotector sobre la incidencia de cáncer ${ }^{17}$. Se podría objetar a estos estudios que no son representativos de la población (se realizaron en profesionales de la salud o en pacientes con Síndrome de Lynch y no parecen por tanto extrapolables), o que la dosis o el periodo de seguimiento parece insuficiente (algunos consideran que es necesario un periodo de observación más largo para detectar una reducción significativa en la incidencia de CCR. Esta observación es con-

\footnotetext{
a Síndrome de Lynch o cáncer colorrectal hereditario no asociado a poliposis (CCHNP): enfermedad hereditaria autosómica dominante causada por mutaciones germinales en genes reparadores del ADN (MLH1, MSH2, MSH6, PMS2), que se caracteriza por el desarrollo precoz de CCR (generalmente antes de los 50 años) y por presentar una elevada tendencia a tener neoplasias extracolónicas (endometrio, ovario, estómago y tracto urinario fundamentalmente) ${ }^{2,4}$
} 
sistente con la evidencia disponible sobre el largo periodo de latencia en la secuencia adenoma-carcinoma). De hecho el mismo grupo de investigación CAPP2, presentó en 2009 en el Congreso de la Organización Europea para el Cáncer y de la Sociedad Europea de Oncología Médica, resultados sorprendentes a más de 5 años de seguimiento en los pacientes con Síndrome de Lynch, que fueron aleatorizados a recibir aspirina, demostrando que reducía en un $50 \%$ el riesgo de CCR en estos pacientes ${ }^{18}$. Estos resultados han sido recientemente publicados en la revista The Lancet $^{19}$. En cuanto a la poliposis adenomatosa familiar ${ }^{\mathrm{b}}$ (PAF) disponemos de estudios que evalúan el efecto protector de los AINE en estos pacientes, pero no propiamente con AAS sino con sulindaco o Coxibs, demostrando un efecto protector de los mismos en la regresión de adenomas $^{20,21}$.

Ensayos clínicos posteriores, como The Nurses'Health Study ${ }^{22}$ o The Health Professionals Follow-up Study ${ }^{23}$ han demostrado un efecto protector frente al CCR en individuos expuestos a mayores dosis de AAS (más de 14 tabletas de AAS a la semana en el caso de The Nurses' Health Study) durante más tiempo (18 años de seguimiento en el caso de The Health Professionals Followup Study). Pero, como se comentará más adelante, dosis mayores de AAS no están exentas de riesgos. Y hay mucha disparidad entre los estudios a la hora de establecer la dosis de AAS o el tiempo de seguimiento necesario.

Por otra parte, considerando que muchos de los efectos secundarios de los AINE se deben a su inhibición de la COX-1, se han desarrollado fármacos que inhiben de forma selectiva la COX-2. Como se ha comentado, la expresión de COX-2 se

b Poliposis adenomatosa familiar (PAF): enfermedad hereditaria autosómica dominante causada por mutaciones germinales en el gen APC (Adenomatous Polyposis Coli), que se caracteriza por la presencia de múltiples pólipos adenomatosos, que pueden aparecer a lo largo de todo el tracto gastrointestinal. Cuando el número de pólipos es superior a 100, hablamos de PAF clásica y cuando oscila entre 20 y 100 , de PAF atenuada ${ }^{2,4}$ ha relacionado con el tamaño y el grado de displasia de los adenomas. De ahí que los estudios con inhibidores de COX-2 (Coxibs) sean especialmente interesantes en pacientes con alta tasa de adenomas, como son los pacientes con poliposis adenomatosa. Efectivamente diversos estudios han mostrado un efecto beneficioso de los Coxibs en su incidencia. Por ejemplo, un ensayo clínico que recogía 77 pacientes con PAF observó una reducción del $28 \%$ de pólipos de recto asociada a la toma de celecoxib ${ }^{21}$; y otro que recogía 21 pacientes con PAF una reducción del $10 \%$ asociada a la toma de rofecoxib ${ }^{24}$.

También se ha observado un efecto beneficioso de los Coxibs en la recurrencia de adenomas en pacientes con historia previa de adenomas. En un ensayo clínico se aleatorizó a pacientes con alto riesgo de adenomas (pacientes con historia de adenomas múltiples o con un adenoma mayor de $5 \mathrm{~mm}$ de diámetro), a recibir $200 \mathrm{mg}$ de celecoxib 2 veces al día, o $400 \mathrm{mg} 2$ veces al día o placebo durante un periodo de 3 años. Comparados con placebo, los que recibieron la mayor dosis de celecoxib, tuvieron un $45 \%$ menos de riesgo de recurrencia de adenomas y los que recibieron la menor dosis un $33 \%$ menos $^{25}$.

\section{RIESGOS Y BENEFICIOS DE LA AAS Y OTROS AINE}

El riesgo de sangrado se explica por bloqueo de la COX-1 (más específica de la AAS y otros inhibidores no selectivos), que inhibe la síntesis de prostaglandina E2 en el epitelio gástrico aumentando así el riesgo de úlcera gástrica y sangrado. Además, los Coxibs y AINE no selectivos inhiben la producción de prostaciclina por el endotelio vascular, incrementando así el riesgo de eventos trombóticos en algunos pacientes $^{26}$.

Las contraindicaciones habituales para el uso de AAS incluyen historia previa de úlceras gastrointestinales, enfermedades hematológicas y reacciones alérgicas.

El riesgo de efectos secundarios aumenta con la edad y la dosis. A partir de los 60 años se incrementa de forma significativa 
el riesgo de hemorragia severa y de úlcera gastrointestinal. Muchos tumores, entre ellos el cáncer de colon, debutan a partir de esa edad, pero no se tiene claro a qué edad sería conveniente comenzar la quimioprofilaxis, ni cuánto tiempo debe mantenerse o cuánto tiempo persiste el efecto protector al suspender el tratamiento. Por su efecto selectivo sobre las plaquetas, dosis pequeñas de AAS previenen eventos trombóticos cardiovasculares, minimizando los riesgos de efectos adversos en el epitelio gástrico. La profilaxis antitrombótica está indicada en pacientes con alto riesgo, pero no en pacientes de riesgo medio, donde el beneficio de la AAS compite con la posibilidad de sangrado $^{27}$. Además no está claro que las pequeñas dosis de AAS que resultan beneficiosas para la prevención cardiovascular, se puedan aplicar de igual modo a las lesiones preneoplásicas o al cáncer.

En relación con los inhibidores selectivos de la COX-2, los estudios sobre Coxibsadenomas tuvieron que interrumpirse por efectos adversos y algunos fármacos fueron retirados del mercado ${ }^{25}$. Sin embargo, en un meta-análisis de 6 ensayos clínicos con celecoxib, se ha visto que puede ser un fármaco relativamente seguro en pacientes con bajo riesgo cardiovascular. Desafortunadamente los pacientes con mayor riesgo en el tratamiento con Coxibs (entiéndase por pacientes de mayor riesgo aquellos con un perfil de obesidad, vida sedentaria, etc.), son también los que tienen más riesgo de desarrollar CCR y por lo tanto los que "a priori" se beneficiarían más de una prevención primaria que en su caso puede suponer mayores efectos secundarios indeseables.

\section{ALTERNATIVAS EN LA PREVENCIÓN DEL CÁNCER COLORRECTAL}

El CCR tiene una peculiaridad que le hace especialmente susceptible de prevención secundaria: presenta una etapa inicial asintomática (correspondiente al adenoma de colon), que en la mayoría de los sujetos es prolongada en el tiempo, lo cual permite desarrollar eficazmente programas de cribado y detección precoz, dirigidos no sólo a la detección del cáncer en estadios curables, sino también a la detección y extirpación eficaz de lesiones premalignas para reducir la incidencia del cáncer en el ámbito clínico habitual ${ }^{28}$. Estos programas de prevención secundaria han demostrado su eficacia, mostrando una reducción significativa de la incidencia y de la mortalidad por CCR, y se están implantando de forma progresiva en las diferentes CCAA de nuestro país ${ }^{3}$. Este hecho deja un margen al adecuado estudio de los posibles fármacos que puedan utilizarse en la quimioprofilaxis, para minimizar los efectos secundarios que se derivarían de la utilización masiva de los mismos en la población general.

\section{CONSIDERACIONES FINALES}

La aspirina y otros AINE se han asociado a una reducción en el riesgo de desarrollar adenomas y CCR. Hasta ahora no está establecida la dosis mínima eficaz, la edad a la que comenzar la quimioprofilaxis y la duración óptima del tratamiento en la profilaxis primaria del CCR. La evidencia disponible en la actualidad sugiere que son necesarias dosis más altas que las aconsejadas en la profilaxis de enfermedades cardiovasculares y que deben mantenerse por un tiempo prolongado.

Conviene sopesar los efectos potenciales del tratamiento a largo plazo con los posibles efectos adversos (toxicidad gastroduodenal, aumento del riesgo cardiovascular o de fenómenos trombóticos), y establecer los grupos de sujetos que mejor se pueden beneficiar de este tipo de prevención limitando el riesgo lo máximo posible. Este planteamiento es todavía más importante considerando que en la actualidad existen alternativas consolidadas que permiten reducir la incidencia y la mortalidad por CCR desde programas de prevención secundaria, que ya se están implantando en la práctica clínica habitual.

De acuerdo con lo expuesto, con la evidencia científica disponible, The US Preventive Services Task Force emitió una declaración de consenso en 2007 sobre el uso de aspirina y AINE para la prevención del CCR. Concluyó, con un grado de recomendación 
1B, que los daños podrían superar a los beneficios en adultos asintomáticos con un riesgo medio de cáncer de colon, incluyendo aquellos con historia familiar de CCR. Hay buena evidencia de que dosis bajas en estos casos, no se asocian a una reducción del riesgo de $\mathrm{CCR}^{7}$.

Por tanto, creemos que está justificada la necesidad de más estudios que evalúen el efecto protector de la AAS u otros AINE como medida preventiva en el cáncer de colon, que definan la dosis mínima eficaz, la edad a la que se debe comenzar el tratamiento, la duración óptima del mismo y las subpoblaciones para las que los beneficios de la quimioprofilaxis superan los posibles efectos adversos.

\section{BIBLIOGRAFÍA}

1. La situación del cáncer en España (19752006) Centro nacional de epidemiología. Instituto de Salud Carlos III. Madrid, junio 2009.

2. Alonso Sánchez A, Moreno S, Valiente A, Artigas M, Pérez-Juana A, Ramos-Arroyo MA. Genetic mechanism in the hereditay predisposition to colorectal cancer. An Sist Sanit Navar 2006; 29: 59-76.

3. Betés MT, Carretero C, Muñoz Navas MA. Evidencia científica en cribado del cáncer colorrectal. Manual de actuación. Madrid: IM \& C; 2006.

4. Zubiarre L, Bujanda Fernández de Pierola L. Aspirina en la prevención del cáncer colorrectal. Gastroenterol Hepatol 2011; 34: 337-345.

5. CHAN AT. NSAIDs (including aspirin): Role in prevention of colorectal cancer. En: Uptodate, Basow, DS (Ed), Update, Waltham, MA, 2009. Sept 2011.

6. Benamouzig R, Uzzan B, Martin A, Deyra J, Little J, GIRARD B et al. Cyclooxygenase-2 expression and recurrence of colorectal adenomas: effect of aspirin chemoprevention. GUT 2010; 59: 622-629.

7. Dubé, C, Rostom, A, Lewin, G, Tsertsvadze A, BARROWMAN N, CODE $\mathrm{C}$ et al. The use of aspirin for primary prevention of colorectal cancer: a sistematic review prepared for the US Preventive Services Task Force. Ann Intern Med 2007; 146: 365-375.

8. Baron JA, Cole BF, SAndler RS, Halle RW, Ahnen $\mathrm{D}$, BRESALIER R et al. A randomized trial of aspirin to prevent colorectal adenomas. N Engl J Med 2003; 348: 891-899.
9. SAndler RS, Halabi S, Baron JA, Budinger S, PasKett E, Keresztes R et al. A randomized trial of aspirin to prevent colorectal adenomas in patients with previous colorectal cancer. $\mathrm{N}$ Engl J Med 2003; 348: 883-890.

10. Logan RF, Grainge MJ, Shepherd VC, Armitage NC, MuIR KR. Aspirin and folic acid for the prevention of recurrent colorectal adenomas. Gastroenterology 2008; 134: 29-38.

11. Benamouzig R, Deyra J, Martin A, Girard B, JuLLIAN E, PIEDNOIR B et al. Daily soluble aspirin and prevention of colorectal adenoma recurrence: one-year results of the APACC trial. Gastroenterology 2003; 125: 328-336.

12. Cole BF, logan RF, Halabi S, Benamouzig R, SANDLER RS, Grainge MJ et al. Aspirin for the chemoprevention of colorectal adenomas: meta-analysis of the randomized trials. J Natl Cancer Inst 2009; 101: 256-266.

13. Cook NR, Lee IM, Gaziano JM, Gordon D, RidKer PM, MANSON JE et al. Low dose of aspirin in the primary prevention of cancer: the Women's Health Study: a randomiced controlled trial. JAMA 2005; 294: 45-55.

14. Gann PH, Manson JE, Glynn RJ, Buring JE, HenNEKENS CH. Low-dose aspirin and incidence of colorectal tumors in a randomized trial. J Natl Cancer Inst 1993; 85: 1220-1224.

15. Stürmer T, Glynn RJ, Lee IM, Manson JE, Buring JE AND HENNEKEns CH. Aspirin use and colorectal cancer: post-trial follow-up data from The Physicians'Health Study. Ann Intern Med 1998; 128: 713-720.

16. Rothwell P, Wilson M, Elwin CE, Norrving B, Algra A, Warlow P et al. Long-term effect of aspirin on colorectal cancer incidence and mortality: 20-year follow-up of five randomised trials. Lancet 2010; 376: 1741-1750.

17. Burn J, Bishop DT, Mecklin JP, Macrae F, MösLEIN G, Olschwang S et al. Effect of aspirin or resistant starch on colorectal neoplasia in the Lynch syndrome. N Eng J Med 2008; 359: 2567-2578.

18. Burn J, Mathers JC, Gerdes AM, Bisgatrd ML, Evans G, Eccles D et al. Cancer occurrence during follow-up of the CAPP2 study -aspirin use for up to four years significantly reduces Lynch syndrome cancers for up to several years after completion of therapy. Hereditary Cancer in Clinical Practice 2010, 8 (Suppl 1): O5.

19. Burn J, Gerdes AM, Macrae F, Mecklin JP, MoesLEIN G, OlschWANG S et al. Long-term effect of aspirin on cancer risk in carriers of hereditary colorectal cancer: an analysis from the 
CAPP2 randomised controlled trial. Lancet 2011; 378: 2081-2087.

20. Giardello FM, Hamilton SR, Krush AJ, PiantadoSi S, Hylind LM, Celano P et al. Treatment of colonic and rectal adenomas with sulindac in familial adenomatous polyposis. N Engl J Med 1993; 328: 1313-1316.

21. Steinbach G, Lynch PM, Phillips RK, Wallace $\mathrm{MH}$, Hawk E, GoRdon GB et al. The effect of Celecoxib, a cyclooxygenase- 2 inhibitor, in familiar adenomatous polyposis. $\mathrm{N}$ Eng $\mathrm{J}$ Med 2000; 342: 1946-1952.

22. Chan AT, Giovannucci EL, Meyerhardt JA, ScherNHAMmer ES, Curhan GC, Fuchs CS. Long-term use of aspirin and nonsteroidal anti-inflammatory drugs and risk of colorectal cancer. JAMA 2005; 294: 914-923.

23. Chan AT, Giovannucci EL, Meyerhardt JA, SchernHAMMER ES, Wu K AND Fuchs CS. Aspirin dose and duration of use and risk of colorectal cancer in men. Gastroenterology 2008; 134: 21-28.

24. Higuchi T, Inana T, Yoshinaga K, Toyooka M, Taketo MM, Sugihara K. A randomized, doubled-blind, placebo-controlled trial of the effects of rofecoxib, a selective cyclooxygenase-2 inhibitor, on rectal polyps in familial adenomatous polyposis patients. Clin Cancer Res 2003; 9: 4756-4760.

25. Bertagnolli MM, Eagle CJ, Zauber AG, Redtson M, Solomon SD, KIm K et al. Celecoxib for the prevention of sporadic colorectal adenomas. N Eng J Med 2006; 355: 873-884.

26. Cuzick J, Otto F, Baron JA, Brown PH, Burn J, Greenwald P et al. Aspirin and non-steroidal anti-inflammatory drugs for cancer prevention: an international consensus statement. Lancet Oncol 2009; 10: 501-507.

27. Antithrombotic Trialists'Collaboration. Collaborative meta-analysis of randomised trials of antiplatelet therapy for prevention of death, myocardial infarction, and stroke in high risk patients. BMJ 2002; 324: 71-86.

28. O'Brien MJ, Winawer SJ, Zauber AG, Sternberg SS, DiAz B, Dickersin GR et al. The National Polyp Study. Patient and polyp characteristics associated with high-grade dysplasia in colorectal adenomas. Gastroenterology 1990; 98: 371-379. 